This item was submitted to Loughborough's Research Repository by the author.

Items in Figshare are protected by copyright, with all rights reserved, unless otherwise indicated.

\title{
Nonlinear disturbance observer-based control for multi-input multi-output nonlinear systems subject to mismatching condition
}

PLEASE CITE THE PUBLISHED VERSION

http://www.tandfonline.com/doi/abs/10.1080/00207179.2012.675520

PUBLISHER

(C) Taylor and Francis

\section{VERSION}

AM (Accepted Manuscript)

\section{LICENCE}

CC BY-NC-ND 4.0

\section{REPOSITORY RECORD}

Yang, Jun, Shihua Li, and Wen-Hua Chen. 2012. "Nonlinear Disturbance Observer-based Control for Multiinput Multi-output Nonlinear Systems Subject to Mismatching Condition". figshare.

https://hdl.handle.net/2134/11207. 
This item was submitted to Loughborough's Institutional Repository (https://dspace.lboro.ac.uk/) by the author and is made available under the following Creative Commons Licence conditions.

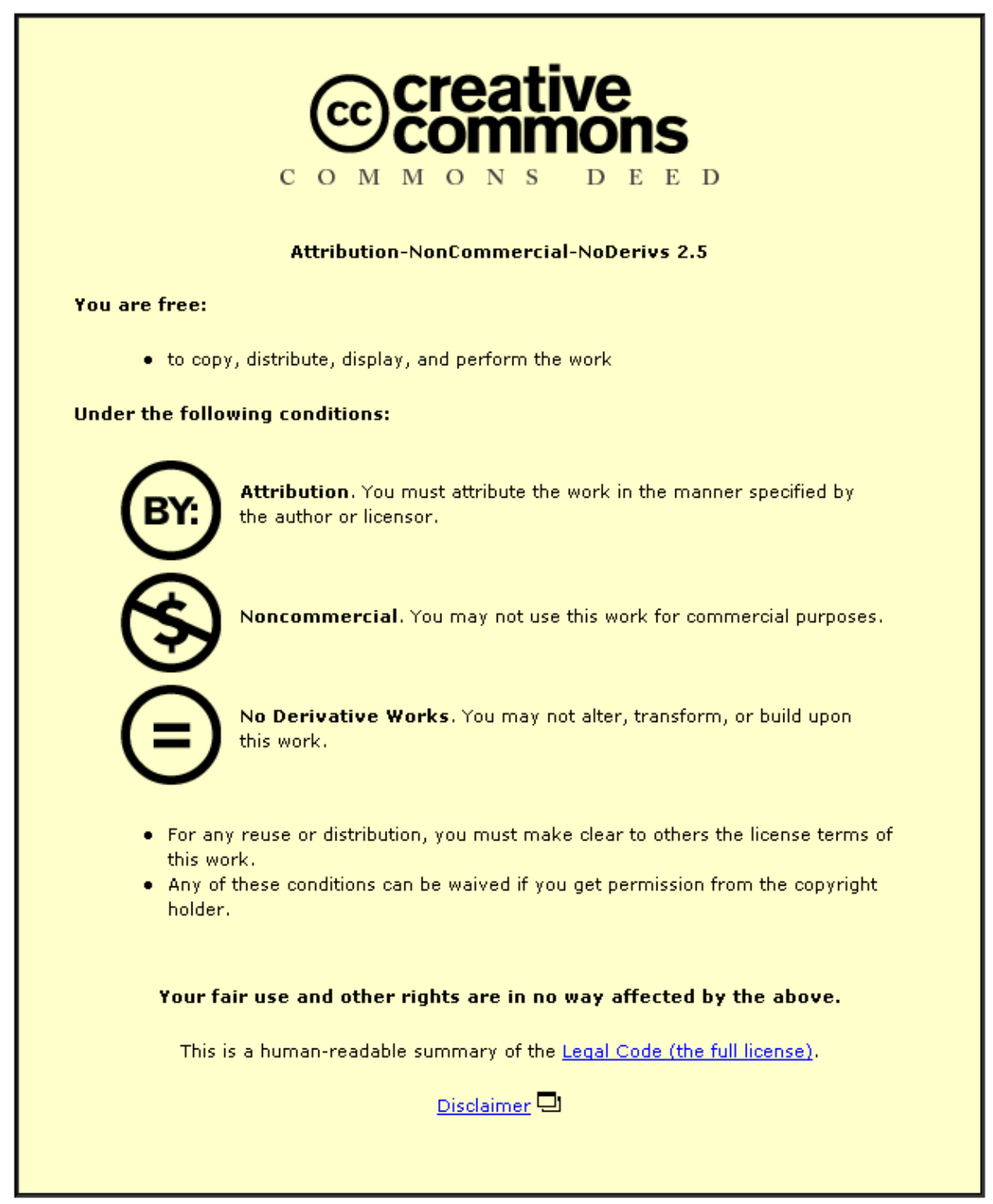

For the full text of this licence, please go to: http://creativecommons.org/licenses/by-nc-nd/2.5/ 


\title{
Nonlinear disturbance observer based control for multi-input multi-output nonlinear systems subject to mismatching condition
}

\author{
Jun Yang ${ }^{a}$, Shihua $\mathrm{Li}^{a *}$ and Wen-Hua Chen ${ }^{b}$ \\ ${ }^{a}$ School of Automation, Southeast University, Key laboratory of Measurement and Control of CSE, \\ Ministry of Education, Nanjing 210096, P.R. China; ${ }^{b}$ Department of Aeronautical and Automotive \\ Engineering, Loughborough University, Leicestershire, LE11 3TU, UK
}

(Received 23 November 2011)

\begin{abstract}
For a multi-input multi-output (MIMO) nonlinear system, the existing disturbance observer based control (DOBC) only provides solutions to those whose disturbance relative degree is higher than or equal to its input relative degree. By designing a novel disturbance compensation gain matrix, a generalized nonlinear disturbance observer based control method is proposed in this article to solve the disturbance attenuation problem of the MIMO nonlinear system with arbitrary disturbance relative degree. It is shown that the disturbances are able to be removed from the output channels by the proposed method with appropriately chosen control parameters. The property of nominal performance recovery, which is the major merit of the DOBCs, is retained with the proposed method. The feasibility and effectiveness of the proposed method are demonstrated by simulation studies of both the numerical and application examples.
\end{abstract}

\begin{abstract}
Keywords: disturbance attenuation; nonlinear disturbance observer; arbitrary disturbance relative degree;
\end{abstract} mismatching condition; multi-input multi-output nonlinear system

\section{Introduction}

Developing efficient control approaches to counteract the adverse effects caused by model uncertainties and external disturbances is an active topic in both the control theory and application community. Many elegant solutions, such as $H_{2} / H_{\infty}$ control, sliding-model control, adaptive control, backstepping control, and so on, have been widely investigated in the literature. Although these methods have gained extensive applications and been proved to be efficient, they mainly focus on the stability(or robust stability) of uncertain systems (Back and Shim 2009), and in general the robustness is achieved at a price of sacrificing the nominal performance (Back and Shim 2008, 2009, Yang, Chen, and Li 2011).

As a practical alternative approach, disturbance observer based control (DOBC) has been proved to be effective in compensating the effects of unknown external disturbances and model uncertainties in control systems and received a great deal of attention in control society, for example, the last decade's development of DOBC can be seen from Chen, Komada, and Fukuda (2000), Chen et al. (2000), Chen (2003), Guo and Chen (2005), Xia et al. (2007), Yoo, Yau, and Gao (2007), Aldeen and Sharma (2008), Back and Shim (2008, 2009), Fujisaki and Befekadu (2009), Han (2009), Shim and Jo (2009), Wei and Guo (2009, 2010), Xia et al. (2011), Yang, Chen, and $\mathrm{Li}$ (2011), and the references therein. The major merit of the DOBC is that the robustness of the closed-loop system is obtained without sacrificing its nominal control performance (Back and Shim 2008).

*Corresponding author. Email: lsh@seu.edu.cn 
In spite of the above excellent feature, most of the existing DOBCs are only available for systems whose disturbance relative degree is higher than or equal to its input relative degree (Chen et al. 1999), which is also referred to as the case that the disturbance satisfies the so-called matching condition (Barmish and Leitmann 1982). Here the matching condition implies that the disturbance act on the system in the same channel as that of the control input. The cases of mismatched and matched disturbance are illustrated by the following typical examples (Marino, Respondek, and V.D. Schaft 1989),

$$
\begin{aligned}
& \left\{\begin{aligned}
\dot{x}_{1} & =\arctan x_{2}+w, \\
\dot{x}_{2} & =u, \\
y & =x_{1},
\end{aligned}\right. \\
& \left\{\begin{aligned}
\dot{x}_{1} & =\arctan x_{2}, \\
\dot{x}_{2} & =u+w, \\
y & =x_{1},
\end{aligned}\right.
\end{aligned}
$$

The disturbance in system (1a) is clearly mismatched one as it appears in different channel from that of the control input, or equivalently, the disturbance relative degree $\nu=1$ is lower than its input relative degree $\sigma=2$. The existing DOBC law $u=K(x)-\hat{w}$ (where $\hat{w}$ is the disturbance estimation) is able to solve the disturbance attenuation problem of system (1b) where the disturbance is matched one, but clearly can not effectively compensate the disturbance in system (1a). It is worth noting that the mismatched disturbances and uncertainties widely exist in practical engineering and applications, such as flight control systems (Chen 2003), and motion control systems (Mohamed 2007, Yang et al. 2011).

Although the disturbance attenuation problems of multi-input multi-output (MIMO) nonlinear systems with mismatched disturbances have been concerned by Wei and Guo (2009, 2010), the problem is not solved entirely by DOBC since only the matched disturbances therein are compensated by DOBC but the mismatched uncertainty attenuation has to resort to other advanced feedback control methods, such as $H_{\infty}$ control (Wei and Guo 2010) and sliding-mode control (Wei and Guo 2009). It is also reported that some constrains on the mismatched uncertainties (such as with bounded $H_{2}$ norm) are required by Wei and Guo $(2009,2010)$.

Recently, several researchers have engaged in the field of attenuating mismatched disturbances via DOBC due to its significance. In She et al. (2008), Maeder, and Morari (2010), Yang et al. (2011), mismatched disturbance attenuation approaches were proposed for linear systems. A generalized linear extended state observer based control method was proposed in Li et al. (2012) to attenuate the mismatched disturbance of a class of single-input single-output (SISO) nonlinear system. By designing a novel disturbance compensation gain, a nonlinear DOBC method was proposed for a special class of SISO nonlinear systems in Yang, Chen, and Li (2011). Yang, Li, and $\mathrm{Yu}$ (2012) proposed a disturbance observer based sliding-mode control method to compensate the mismatched uncertainties of SISO nonlinear systems. However, disturbance attenuation of multi-input multi-output (MIMO) nonlinear systems subject to mismatching condition is still an unsolved problem in the DOBC community.

In this article, a generalized nonlinear disturbance observer based control (NDOBC) is proposed to compensate the effects caused by mismatched disturbances of MIMO nonlinear systems. By properly designing a disturbance compensation gain matrix, it is proved that the mismatched disturbances could be eliminated from the output channels. The major merit of proposed method is that the promising disturbance attenuation performance is achieved without sacrificing its nominal control performance of the closed-loop system, which is referred to as nominal performance recovery. The effectiveness of the proposed method is validated by simulation studies of both the numerical and application example. 


\section{Problem formulation and preliminaries}

\subsection{Problem formulation}

Consider a MIMO nonlinear system depicted by

$$
\left\{\begin{array}{l}
\dot{x}=f(x)+g(x) u+p(x) w \\
y=h(x)
\end{array}\right.
$$

where $x=\left[x_{1}, \ldots, x_{n}\right]^{T} \in R^{n}, u=\left[u_{1}, \ldots, u_{m}\right]^{T} \in R^{m}, w=\left[w_{1}, \ldots, w_{n}\right]^{T} \in R^{n}$ and $y=$ $\left[y_{1}, \ldots, y_{m}\right]^{T} \in R^{m}$ denote the state, input, disturbance and output vectors, respectively. $f(x)$, $g(x)=\left[g_{1}(x), \ldots, g_{m}(x)\right], p(x)=\left[p_{1}(x), \ldots, p_{n}(x)\right]$, and $h(x)=\left[h_{1}(x), \ldots, h_{m}(x)\right]^{T}$ are smooth vector or matrix fields on $R^{n}$. Without loss of generality, it is supposed that the equilibrium of system (2) in the absence of disturbances is $x_{0}=0$. The standard Lie derivative notation is used in this paper, stated as follows (Isidori 1995).

Definition 1 (Isidori 1995): The vector relative degree from the control inputs to the outputs of system $(2)$ is $\left(\sigma_{1}, \ldots, \sigma_{m}\right)$ at the equilibrium $x_{0}$ if $L_{g_{j}} L_{f}^{k} h_{i}(x)=0(1 \leq j \leq m, 1 \leq i \leq m)$ for all $k<\sigma_{i}-1$, and for all $x$ in a neighborhood of $x_{0}$, and the $m \times m$ matrix

$$
A(x)=\left[\begin{array}{cccc}
L_{g_{1}} L_{f}^{\sigma_{1}-1} h_{1} & L_{g_{2}} L_{f}^{\sigma_{1}-1} h_{1} & \cdots & L_{g_{m}} L_{f}^{\sigma_{1}-1} h_{1} \\
L_{g_{1}} L_{f}^{\sigma_{2}-1} h_{2} & L_{g_{2}} L_{f}^{\sigma_{2}-1} h_{2} & \cdots & L_{g_{m}} L_{f}^{\sigma_{2}-1} h_{2} \\
\vdots & \vdots & \ddots & \vdots \\
L_{g_{1}} L_{f}^{\sigma_{m}-1} h_{m} & L_{g_{2}} L_{f}^{\sigma_{m}-1} h_{m} & \cdots & L_{g_{m}} L_{f}^{\sigma_{m}-1} h_{m}
\end{array}\right]
$$

nonsingular at $x=x_{0}$. For simplicity, it is referred to as the input relative degree (IRD). Similarly, the disturbance relative degree (DRD) at $x_{0}$ is defined as $\left(\nu_{1}, \ldots, \nu_{m}\right)$.

Remark 1 (Chen et al. 1999): The existing nonlinear disturbance observer based control (NDOBC) is only available for nonlinear system (2) whose DRDs are higher than or equal to its IRDs, i.e., $\nu_{i} \geq \sigma_{j}$ for all $1 \leq i \leq m$ and $1 \leq j \leq m$.

Remark 2: When it comes to the case that some DRD of nonlinear system (2) is strictly lower than some IRD, i.e., $\nu_{i}<\sigma_{j}$ for some $i, j \in\{1, \ldots, m\}$, the disturbances will inevitably affect the states regardless of what feedback-based control method is employed. In this case, the most sensible design goal would be to find a control method such that the disturbances do not affect the interested output variables (at least in steady-state). As pointed in Remark 1, the existing NDOBC is no longer available for this case, which motivates further research on generalized NDOBC for system (2) with arbitrary DRDs.

\subsection{Nonlinear disturbance observer}

The nonlinear disturbance observer (NDOB) proposed by Chen et al. (1999) provides an adequate way to estimate the disturbances in system (2), given by

$$
\left\{\begin{array}{l}
\dot{z}_{w}=-l(x)\left[p(x)\left(\lambda(x)+z_{w}\right)+f(x)+g(x) u\right] \\
\hat{w}=z_{w}+\lambda(x)
\end{array}\right.
$$

where $\hat{w}=\left[\hat{w}_{1}, \ldots, \hat{w}_{n}\right]^{T}$ and $z_{w}$ are the estimation vector of the disturbance vector $w$ and the internal state vector of the nonlinear observer, respectively. $\lambda(x)$ is a nonlinear function to be designed. The observer gain $l(x)$ is designed as

$$
l(x)=\frac{\partial \lambda(x)}{\partial x}
$$


The disturbance estimation error of (4) for system (2) is obtained by

$$
\dot{e}_{w}(t)=-l(x) p(x) e_{w}(t)+\dot{w},
$$

where the estimation error is defined as $e_{w}=w-\hat{w}$.

Assumption 1: The derivatives of the disturbances in system (2) are bounded, i.e., $\|\dot{w}(t)\|<\infty$. This is a general assumption made for the disturbance estimation. All continuous and bounded disturbances satisfies this assumption.

Lemma 1 (Chen and Guo 2004): Suppose that Assumption 1 is satisfied. The disturbance estimation error system (5) is locally input-to-state stable (ISS) if the observer gain $l(x)$ is chosen such that

$$
\dot{e}_{w}(t)+l(x) p(x) e_{w}(t)=0
$$

is asymptotically stable.

Assumption 2: The disturbances in (2) are bounded and satisfy $\lim _{t \rightarrow \infty} \dot{w}(t)=0$. This assumption is made for steady-state analysis of disturbance estimation and compensation.

Lemma 2: Suppose that Assumption 2 is satisfied. The disturbance estimation $\hat{w}$ of NDOB (4) can asymptotically estimate the disturbance $w$ in system (2) if the observer gain $l(x)$ is chosen such that (6) is asymptotically stable. The proof of this lemma can be easily derived by combining the result of Lemma 1 with the ISS definition in Khalil (1996).

\section{Generalized nonlinear disturbance observer based control}

In order to present our main result, the disturbance decoupling for MIMO nonlinear systems with arbitrary DRDs is solved firstly.

\subsection{Disturbance decoupling}

Disturbance decoupling control, which could attenuate the disturbances from the output channels, is an effective disturbance attenuation method. The disturbance decoupling control approach has been developed for linear systems (Wonham 1985) and also studied for nonlinear systems (Isidori 1995). However, the existing disturbance decoupling methods are only available for systems under matched disturbances. In this part, a novel disturbance decoupling control approach is proposed to attenuate the mismatched disturbances of the MIMO nonlinear systems.

Assumption 3: The distribution $G=\operatorname{span}\left\{g_{1}, \ldots, g_{m}\right\}$ of the MIMO nonlinear system (2) is involutive. This is a necessary condition for state feedback linearisation of MIMO nonlinear systems (Isidori 1995).

Disturbance decoupling problem for a MIMO nonlinear system with arbitrary DRDs is solved by Theorem 3.1 .

Theorem 3.1: Consider a MIMO nonlinear system (2) with disturbances have arbitrary DRDs satisfying the condition in Assumptions 2-3. A disturbance decoupling control law which can compensate the disturbances in the output channels of (2) in steady state is given by

$$
u=A^{-1}(x)[-b(x)+v+\Gamma(x) w]
$$


where $A(x)$ is the same as that in Eq. (3),

$$
\Gamma(x)=\left[\begin{array}{cccc}
\gamma_{11}(x) & \gamma_{12}(x) & \cdots & \gamma_{1 n}(x) \\
\gamma_{21}(x) & \gamma_{22}(x) & \cdots & \gamma_{2 n}(x) \\
\vdots & \vdots & \ddots & \vdots \\
\gamma_{m 1}(x) & \gamma_{m 2}(x) & \cdots & \gamma_{m n}(x)
\end{array}\right], b(x)=\left[\begin{array}{c}
b_{1}(x) \\
b_{2}(x) \\
\vdots \\
b_{m}(x)
\end{array}\right], v=\left[\begin{array}{c}
v_{1} \\
v_{2} \\
\vdots \\
v_{m}
\end{array}\right]
$$

with

$$
\begin{gathered}
\gamma_{i j}(x)=-\sum_{k=0}^{\sigma_{i}-2} c_{k+1}^{i} L_{p_{j}} L_{f}^{k} h_{i}-L_{p_{j}} L_{f}^{\sigma_{i}-1} h_{i},(i=1,2, \ldots, m ; j=1,2, \ldots, n), \\
b_{i}(x)=L_{f}^{\sigma_{i}} h_{i}, v_{i}=-\sum_{k=0}^{\sigma_{i}-1} c_{k}^{i} L_{f}^{k} h_{i},(i=1,2, \ldots, m),
\end{gathered}
$$

where parameters $c_{k}^{i}\left(i=1,2, \ldots, m ; k=0,1, \ldots, \sigma_{i}-1\right)$ have to be designed such that the polynomials

$$
p_{0}^{i}(s)=c_{0}^{i}+c_{1}^{i} s+\cdots+c_{\sigma_{i}-1}^{i} s^{\sigma_{i}-1}+s^{\sigma_{i}},
$$

are Hurwitz stable.

Proof: The partially feedback linearisation of the nonlinear system (2) in the absence of disturbances is addressed firstly for the case of $\sigma=\sigma_{1}+\sigma_{2}+\ldots+\sigma_{m}<n$ since it is a general case (the case $\sigma=n$ is a special case and very straightforward)(Isidori 1995). Under the condition of Assumption 3, a new group of coordinate transformation which can feedback linearise system (2) is defined as (Isidori 1995)

$$
\Phi(x)=\left[\begin{array}{l}
\xi \\
\eta
\end{array}\right],
$$

where $\xi=\left[\xi^{1^{T}}, \ldots, \xi^{m T}\right]^{T}$ with

$$
\xi^{i}=\left[\begin{array}{c}
\xi_{1}^{i} \\
\xi_{2}^{i} \\
\vdots \\
\xi_{\sigma_{i}}^{i}
\end{array}\right]=\left[\begin{array}{c}
h_{i}(x) \\
L_{f} h_{i}(x) \\
\vdots \\
L_{f}^{\sigma_{i}-1} h_{i}(x)
\end{array}\right]
$$

for $1 \leq i \leq m$, and $\eta=\left[\eta_{\sigma+1}(x), \ldots, \eta_{n}(x)\right]^{T}$ is selected such that the mapping $\Phi(x)$ has a jacobian matrix which is nonsingular and $L_{g_{j}} \eta_{i}(x)=0$ for all $\sigma+1 \leq i \leq n$ and $1 \leq j \leq m$.

The description of the system (2) under the new coordinates $\Phi(x)$ is then expressed as $m+1$ 
subsystems, and the $i$ th one of the first $m$ subsystems can be represented as

$$
\Pi_{i}:\left\{\begin{aligned}
\dot{\xi}_{1}^{i} & =\xi_{2}^{i}+\sum_{k=1}^{n} L_{p_{k}} h_{i} w_{k}, \\
\dot{\xi}_{2}^{i} & =\xi_{3}^{i}+\sum_{k=1}^{n} L_{p_{k}} L_{f} h_{i} w_{k}, \\
& \vdots \\
\dot{\xi}_{\sigma_{i}}^{i} & =L_{f}^{\sigma_{i}} h_{i}(x)+\sum_{k=1}^{m} L_{g_{k}} L_{f}^{\sigma_{i}-1} h_{i} u_{k}+\sum_{k=1}^{n} L_{p_{k}} L_{f}^{\sigma_{i}-1} h_{i} w_{k}, \\
y_{i} & =\xi_{1}^{i},
\end{aligned}\right.
$$

for all $1 \leq i \leq m$. The last subsystem has the following form

$$
\Pi_{m+1}: \dot{\eta}=q(\xi, \eta)+r(\xi, \eta) w
$$

Let $\underline{\xi}=\left[\xi_{\sigma_{1}}^{1}, \ldots, \xi_{\sigma_{m}}^{m}\right]^{T}$, by collecting the last state equation in (11) for all $1 \leq i \leq m$ to formulate a new vector, it is obtained that

$$
\underline{\dot{\xi}}=b(x)+A(x) u+D(x) w
$$

where

$$
D(x)=\left[\begin{array}{cccc}
L_{p_{1}} L_{f}^{\sigma_{1}-1} h_{1} & L_{p_{2}} L_{f}^{\sigma_{1}-1} h_{1} & \cdots & L_{p_{n}} L_{f}^{\sigma_{1}-1} h_{1} \\
L_{p_{1}} L_{f}^{\sigma_{2}-1} h_{2} & L_{p_{2}} L_{f}^{\sigma_{2}-1} h_{2} & \cdots & L_{p_{n}} L_{f}^{\sigma_{2}-1} h_{2} \\
\vdots & \vdots & \ddots & \vdots \\
L_{p_{1}} L_{f}^{\sigma_{m}-1} h_{m} & L_{p_{2}} L_{f}^{\sigma_{m}-1} h_{m} & \cdots & L_{p_{n}} L_{f}^{\sigma_{m}-1} h_{m}
\end{array}\right]
$$

Substituting the control law (7) into Eq. (13), yields

$$
\underline{\dot{\xi}}=v+\Gamma(x) w+D(x) w
$$

or an equivalent expression of

$$
\dot{\xi}_{\sigma_{i}}^{i}=v_{i}+\sum_{k=1}^{n}\left(\gamma_{i k}+L_{p_{k}} L_{f}^{\sigma_{i}-1} h_{i}\right) w_{k}
$$

Combining Eq. (15) with Eq. (11), the subsystem $\Pi_{i}$ can be rewritten as

$$
\Pi_{i}:\left\{\begin{array}{l}
\dot{\xi}^{i}=\underline{A}^{i} \xi^{i}+\underline{D}^{i}(x) w \\
y_{i}=\bar{C}^{i} \xi^{i}
\end{array}\right.
$$

for $1 \leq i \leq m$, where

$$
\underline{A}^{i}=\left[\begin{array}{ccccc}
0 & 1 & 0 & \cdots & 0 \\
0 & 0 & 1 & \cdots & 0 \\
\vdots & \vdots & \vdots & \ddots & \vdots \\
0 & 0 & 0 & \cdots & 1 \\
-c_{0}^{i} & -c_{1}^{i} & -c_{2}^{i} & \cdots & -c_{\sigma_{i}-1}^{i}
\end{array}\right]
$$




$$
\begin{gathered}
\underline{D}^{i}(x)=\left[\underline{d}_{1}^{i}, \ldots, \underline{d}_{n}^{i}\right], \\
\underline{d}_{j}^{i}=\left[\begin{array}{c}
L_{p_{j}} h_{i} \\
L_{p_{j}} L_{f} h_{i} \\
\vdots \\
-\sum_{k=0}^{\sigma_{i}-2} c_{k+1}^{i} L_{p_{j}} L_{f}^{k} h_{i}
\end{array}\right], 1 \leq j \leq n, \\
C^{i}=[1,0, \ldots, 0]_{1 \times \sigma_{i}} .
\end{gathered}
$$

By simple matrix calculations, it can be verified that

$$
C^{i}\left(\underline{A}^{i}\right)^{-1} \underline{D}^{i}(x)=0,
$$

for all $x \in R^{n}$. To this end, it follows from Eqs. (16) and (17) that

$$
y_{i}=C^{i}\left(\underline{A}^{i}\right)^{-1}\left[\dot{\xi}^{i}-\underline{D}^{i}(x) w\right]=C^{i}\left(\underline{A}^{i}\right)^{-1} \dot{\xi}^{i} .
$$

It can be concluded from Eq. (18) that the disturbances are compensated from the output channel of system (2) with arbitrary DRDs in a static way. Particularly, if the system has a steady state, it can be obtained that the steady state value of the output $y_{i}$ satisfies $y_{i s}=0$.

\subsection{Generalized nonlinear disturbance observer based control}

\subsubsection{Control law design}

The proposed generalized NDOBC law is constructed by replacing the disturbance vector $w$ in the disturbance decoupling control law (7) with the disturbance estimation vector $\hat{w}$ of the NDOB (4).

Theorem 3.2 : Consider a MIMO nonlinear system (2) with disturbances have arbitrary DRDs satisfying Assumptions 2-3, and also suppose that the observer gain $l(x)$ of the NDOB (4) is selected such that system (6) is asymptotically stable. A generalized NDOBC law which compensates the disturbances in the output channels of system (2) is given by

$$
u=A^{-1}(x)[-b(x)+v+\Gamma(x) \hat{w}],
$$

where $\hat{w}$ is the disturbance estimation vector by the $N D O B(4), A(x), b(x), v$, and $\Gamma(x)$ are the same as those defined in Eqs. (3) and (7).

Proof: Under the new group of coordinate transformation $\Phi(x)$ in (9), the nonlinear system (2) has been reformulated as those in Eqs. (11)-(13). Substituting the control law (19) into Eq. (13), gives

$$
\underline{\dot{\xi}}=v+\Gamma(x) \hat{w}+D(x) w
$$

or equivalently expressed as

$$
\dot{\xi}_{\sigma_{i}}^{i}=v_{i}+\sum_{k=1}^{n}\left(\gamma_{i k}+L_{p_{k}} L_{f}^{\sigma_{i}-1} h_{i}\right) w_{k}+\sum_{k=1}^{n} \gamma_{i k}\left(\hat{w}_{k}-w_{k}\right),
$$


for $1 \leq i \leq m$. Combining Eq. (21) with Eq. (11), the subsystem $\Pi_{i}$ is rewritten as

$$
\Pi_{i}:\left\{\begin{array}{l}
\dot{\xi}^{i}=\underline{A}^{i} \xi^{i}+\underline{D}^{i}(x) w-\underline{\Gamma}^{i}(x) e_{w}, \\
y_{i}=\bar{C}^{i} \xi^{i}
\end{array}\right.
$$

for $1 \leq i \leq m$, where $\underline{A}^{i}, \underline{D}^{i}(x), C^{i}$ have been given in Eq. (16), and

$$
\underline{\Gamma}^{i}(x)=\left[\begin{array}{cccc}
0 & 0 & \cdots & 0 \\
\vdots & \vdots & \ddots & \vdots \\
0 & 0 & \cdots & 0 \\
\gamma_{i 1}(x) & \gamma_{i 2}(x) & \cdots & \gamma_{i n}(x)
\end{array}\right]
$$

It follows from Eqs. (22) and (17) that

$$
\begin{aligned}
y_{i} & =C^{i}\left(\underline{A}^{i}\right)^{-1}\left[\dot{\xi}^{i}-\underline{D}^{i}(x) w+\underline{\Gamma}^{i}(x) e_{w}\right] \\
& =C^{i}\left(\underline{A}^{i}\right)^{-1}\left[\dot{\xi}^{i}+\underline{\Gamma}^{i}(x) e_{w}\right] .
\end{aligned}
$$

Eq. (23) implies that the disturbances are decoupled from the output channel of the nonlinear system (2) in an asymptotical way. It is derived from Eq. (23) that

$$
\begin{aligned}
\left|y_{i}\right| & \leq\left\|C^{i}\left(\underline{A}^{i}\right)^{-1}\right\| \cdot\left(\left\|\dot{\xi}^{i}\right\|+\left\|\underline{\Gamma}^{i}(x)\right\| \cdot\left\|e_{w}\right\|\right) \\
& \leq\left\|C^{i}\left(\underline{A}^{i}\right)^{-1}\right\| \cdot\left(\left\|\dot{\xi}^{i}\right\|+L_{1}\left\|e_{w}\right\|\right),
\end{aligned}
$$

where $L_{1}$ is a constant such that $\left\|\underline{\Gamma}^{i}(x)\right\| \leq L_{1}$ over the bounded area of $x$. Since Lemma 2 shows that $\lim _{t \rightarrow \infty} e_{w}(t)=0$, the following condition which represent the steady state of the output can be obtained by taking limits on both sides of Eq. (24),

$$
\begin{aligned}
\lim _{t \rightarrow \infty}\left|y_{i}(t)\right| \leq & \left\|C^{i}\left(\underline{A}^{i}\right)^{-1}\right\| \\
& \times\left(\lim _{t \rightarrow \infty}\left\|\dot{\xi}^{i}(t)\right\|+L_{1} \lim _{t \rightarrow \infty}\left\|e_{w}(t)\right\|\right) \\
= & 0 .
\end{aligned}
$$

This completes the proof.

Remark 3: If the disturbances in (2) are matched ones, the result in Theorem 3.1 reduces to the existing DOBC. Particularly, $g(x)=p(x)$ implies that $\Gamma(x)=-A(x)$, and the resultant control law (19) becomes $u=A^{-1}(x)[-b(x)+v]-\hat{w}$, which is the same as that of the existing method (Chen et al. 1999).

Remark 4: In the absence of disturbances, the disturbance estimate by (4) satisfies $\hat{w}(t) \equiv 0$ if the initial state of the NDOB (4) is set as $z_{w}(0)=-\lambda(x(0))$. In this case, the control performance under the proposed control law (19) recovers to that under the baseline feedback control law, which implies that the property of nominal performance recovery is obtained by the proposed NDOBC.

\subsubsection{Stability of the closed-loop system}

Assumption 4: The zero dynamics of the nonlinear system (2) in the absence of disturbances, i.e., $\dot{x}=f(x)+g(x) u$, under the baseline feedback control law $u=A^{-1}(x)[-b(x)+v]$ are locally asymptotically stable at $x_{0}$. This is a necessary condition for stability analysis of the closed-loop MIMO nonlinear system under the baseline controller (Isidori 1995). 
Lemma 3 (Isidori 1995): Consider a system

$$
\left\{\begin{array}{l}
\dot{\xi}=\bar{A} \xi+p(\xi, \eta) \\
\dot{\eta}=q(\xi, \eta)
\end{array}\right.
$$

and suppose that $p(0, \eta)=0$ for all $\eta$ near 0 and $\frac{\partial p}{\partial \xi}(0,0)=0$. If $\dot{\eta}=f(0, \eta)$ has an asymptotically stable equilibrium at $\eta=0$ and the eigenvalues of $\bar{A}$ all have negative real part, then the system (26) has an asymptotically stable equilibrium at $(\xi, \eta)=(0,0)$.

The stability of the resultant closed-loop system is established by Theorem 3.3.

Theorem 3.3: The nonlinear system (2) under the proposed NDOBC law (19) is locally ISS around $x_{0}$ if the following conditions are satisfied:

(i). Assumptions 1, 3-4 are satisfied;

(ii). the control parameters $c_{k}^{i}$ in the control law (19) are selected such that the polynomials $p_{0}^{i}(s)$ in (8) are Hurwitz stable;

(iii). the observer gain $l(x)$ is selected such that the system (6) is asymptotically stable;

(iv). the disturbance compensation gain is selected such that $g(x) A^{-1}(x) \Gamma(x)+p(x)$ is continuously differentiable around $x_{0}$.

Proof: Taking into account the observer dynamics (5) and substituting the proposed NDOBC law (19) into the system (2), the augmented closed-loop system is obtained, described by

$$
\left\{\begin{aligned}
\dot{x} & =F\left(x, e_{w}, w\right) \\
\dot{e}_{w} & =G\left(e_{w}, \dot{w}\right)
\end{aligned}\right.
$$

where

$$
\begin{gathered}
F\left(x, e_{w}, w\right)=f(x)+g(x) A^{-1}(x)\left[-b(x)+v-\Gamma(x) e_{w}\right] \\
+\left[g(x) A^{-1}(x) \Gamma(x)+p(x)\right] w, \\
G\left(e_{w}, \dot{w}\right)=-l(x) p(x) e_{w}+\dot{w} .
\end{gathered}
$$

Under the new coordinate transformations $\left(\xi^{T}, \eta^{T}\right)^{T}$, the closed-loop system consisting of the system $\dot{x}=f(x)+g(x) u$ and the baseline feedback control law $u=A^{-1}(x)[-b(x)+v]$ is given by

$$
\left\{\begin{array}{l}
\dot{\xi}=\bar{A} \xi \\
\dot{\eta}=q(\xi, \eta)
\end{array}\right.
$$

where $\bar{A}=\operatorname{diag}\left\{\underline{A}^{1}, \ldots, \underline{A}^{m}\right\}$. It is easy to verify that the closed-loop system (30) has the form of system (26). The Assumption 4 and the condition (ii) imply that the conditions of Lemma 3 are satisfied for system (30), and it can be concluded from the lemma that the system (30) (or equivalently the system $\dot{x}=F(x, 0,0))$ has an asymptotically stable equilibrium at $(\xi, \eta)=(0,0)$ (or $x=0$ ).

Let $X=\left[x^{T}, e_{w}^{T}\right]^{T}$, the augmented closed-loop system (27) is rewritten as

$$
\dot{X}=\underline{F}(X)+\underline{G}(X) \underline{w},
$$

where

$$
\underline{F}(X)=\left[\begin{array}{c}
F\left(x, e_{w}, 0\right) \\
G\left(e_{w}, 0\right)
\end{array}\right], \underline{G}(X)=\left[\begin{array}{cc}
g(x) A^{-1}(x) \Gamma(x)+p(x) & 0 \\
0 & I_{n \times n}
\end{array}\right], \underline{w}=\left[\begin{array}{c}
w \\
\dot{w}
\end{array}\right] .
$$


(a)

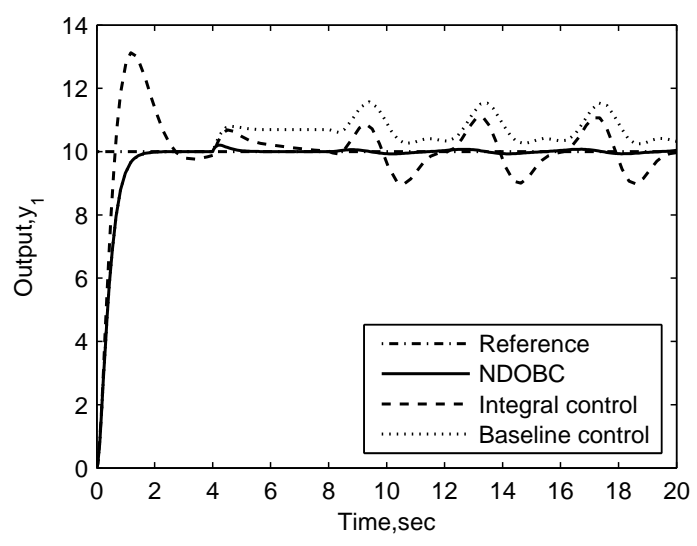

(b)

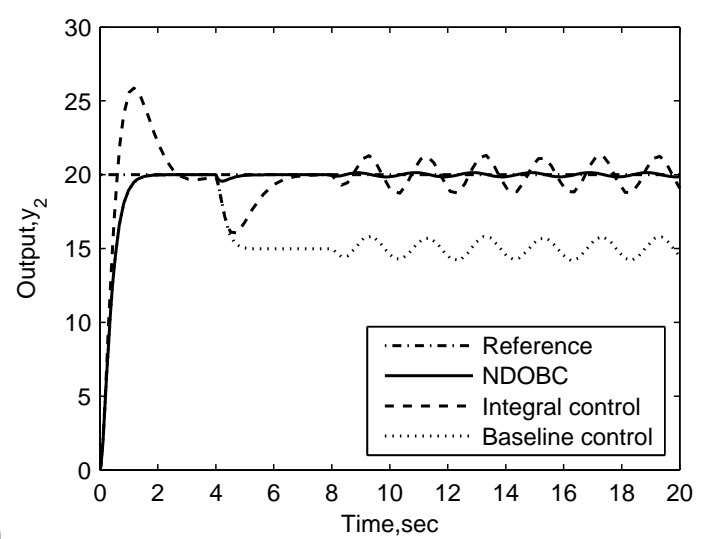

Figure 1. Output response curves of system (32) in the presence of unknown external disturbances under the control laws (19) (solid line), (33) (dotted line) and (34) (dashed line). The reference signals are denoted by dash-dotted line.

According to the theorem of the asymptotic stability of cascade-connected systems (see Corollary 10.3.2 in Isidori (1999)), the system $\dot{X}=\underline{F}(X)$ is locally asymptotically stable at $X=0$.

Combining the above result with condition (iv), it follows from Lemma 5.4 in Khalil (1996) that the augmented closed-loop system (31) is locally ISS, which completes the proof of this theorem.

\section{Simulation stuides}

\subsection{A numerical example}

Consider a MIMO nonlinear system depicted by

$$
\left\{\begin{array}{l}
\dot{x}_{1}=-x_{1}+x_{1} x_{2}+x_{3}+w_{1}, \\
\dot{x}_{2}=\sin x_{1}+x_{2}^{2}+x_{4}+w_{2}, \\
\dot{x}_{3}=x_{4}+u_{1} \\
\dot{x}_{4}=u_{2} \\
\dot{y}_{1}=x_{1}, y_{2}=x_{2}
\end{array}\right.
$$

The IRDs and DRDs of system (32) are calculated as $\left(\sigma_{1}, \sigma_{2}\right)=(2,2)$, and $\left(\nu_{1}, \nu_{2}\right)=(1,1)$, respectively. This is the case where the DRDs of the system are strictly lower than the IRDs, and the conditions in Remark 1 are not satisfied.

The NDOBC law for system (32) is constructed by (19) with some derivative calculations. The control parameters are selected as $c_{0}^{1}=20, c_{1}^{1}=9, c_{0}^{2}=24, c_{1}^{2}=10$. The gain matrix in the NDOB (4) is selected as $\lambda(x)=\left[50 x_{1}, 50 x_{2}\right]^{T}$. To evaluate the effectiveness of the proposed method, both the baseline control and integral control methods are employed in the simulation studies for the purpose of comparison. The baseline controller is given as

$$
u=A^{-1}(x)[-b(x)+v] .
$$

The integral control is designed as

$$
u=A^{-1}(x)\left[-b(x)+v+u_{\text {int }}\right]
$$

where $u_{\text {int }}=\left[-c_{i}^{1} \int\left(y_{1}-r_{1}\right) d t,-c_{i}^{2} \int\left(y_{2}-r_{2}\right) d t\right]^{T}$, with $r_{1}$ and $r_{2}$ the reference signals, $c_{i}^{1}$ and $c_{i}^{2}$ are the integral coefficients, which are chosen as $c_{i}^{1}=25$ and $c_{i}^{2}=30$ for simulation studies.

The reference signals are selected as $r_{1}=10$ and $r_{2}=20$, respectively. The unknown external 
(a)

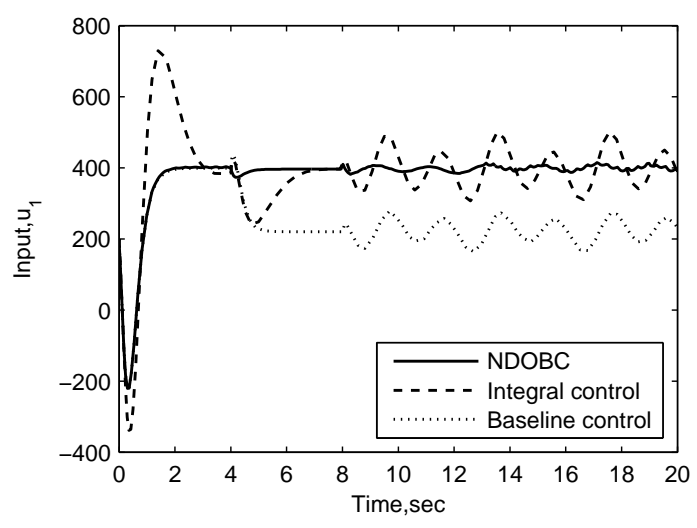

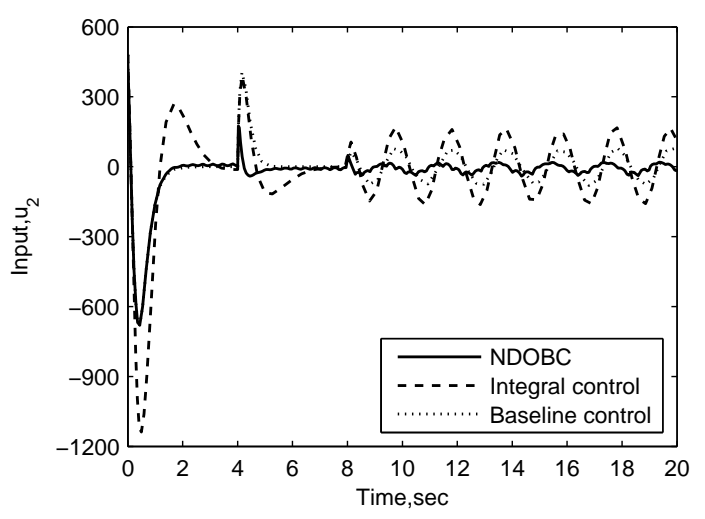

Figure 2. Input profiles of system (32) in the presence of unknown external disturbances under the control laws (19) (solid line), (33) (dotted line) and (34) (dashed line).

disturbances,

$$
\left\{\begin{array}{lrl}
w_{1}(t)=0, w_{2}(t)=0, & \text { for } 0 \leq t<4 \\
w_{1}(t)=2, w_{2}(t)=-3, & \text { for } 4 \leq t<8 \\
w_{1}(t)=0.6 \sin \left(\frac{\pi}{2} t\right)+2, w_{2}(t)=-0.8 \sin \left(\pi t+\frac{\pi}{2}\right)-3, & \text { for } 8 \leq t \leq 20
\end{array}\right.
$$

are considered to be imposed on system (32).

The output response curves of system (32) subject to disturbances (35) under the three controllers are shown in Fig. 1. The corresponding input profiles are shown by Fig. 2. It is observed from Fig. 1 that the baseline controller achieves excellent tracking performance, but quite poor disturbance attenuation performance. The integral control method improves the disturbance rejection performance to some extent, however, it brings certain undesired control performance, such as unsatisfactory overshoots. This implies that the disturbance rejection performance of integral control is achieved at the price of sacrificing its nominal control performance.

A brief observation of Fig. 1 shows that the proposed NDOBC method exhibits promising disturbance attenuation and reference tracking performance. It is also observed from Figs. 1 and 2 that the response curves under the NDOBC are overlapped with those under the baseline control method during the first 4 seconds when there are no disturbances imposed on the system, which is a clear evidence that the property of nominal performance recovery is retained for the NDOBC method.

\subsection{Application to a permanent magnet synchronous motor}

The mathematical model of the permanent magnet synchronous motor (PMSM) in the rotor reference frame is depicted by Krishnan (2001)

$$
\left\{\begin{array}{l}
\frac{d \omega}{d t}=\frac{K_{t}}{J} i_{q}-\frac{B}{J} \omega+d_{\omega} \\
\frac{d i_{d}}{d t}=-\frac{R_{s}}{L_{d}} i_{d}+n_{p} \omega i_{q}+\frac{1}{L_{d}} u_{d}+d_{i_{d}} \\
\frac{d i_{q}}{d t}=-\frac{R_{s}}{L_{q}} i_{q}-n_{p} \omega i_{d}-\frac{n_{p} \phi_{v}}{L_{q}} \omega+\frac{1}{L_{q}} u_{q}+d_{i_{q}}
\end{array}\right.
$$

where $\omega$ the rotor speed, $i_{d}$ and $i_{q}$ the $d$-axis and $q$-axis stator currents, $u_{d}$ and $u_{q}$ the $d$-axis and $q$-axis stator voltages, $R_{s}, L_{d}$, and $L_{q}$ the stator resistance, $d$-axis and $q$-axis inductances, $n_{p}$ the number of pole pairs, $\phi_{v}$ the rotor flux, $J$ the moment of inertia, $B$ the viscous friction coefficient, and $K_{t}=3 n_{p} \phi_{v} / 2$, respectively. $d_{\omega}, d_{i_{d}}$, and $d_{i_{q}}$ denote the unknown disturbances 
which lump all the influence of parameter variations and external disturbances, given by

$$
\begin{gathered}
d_{\omega}=\frac{3 n_{p} \Delta \phi_{v}}{2 J} i_{q}-\frac{\Delta B}{J} \omega-\frac{\Delta J}{J} \frac{d \omega}{d t}-\frac{1}{J} T_{L}, \\
d_{i_{d}}=\frac{\Delta L_{d} n_{p}}{L_{d}} \omega i_{q}-\frac{\Delta R_{s}}{L_{d}} i_{d}-\frac{\Delta L_{d}}{L_{d}} \frac{d i_{d}}{d t}, \\
d_{i_{q}}=-\frac{\Delta L_{q} n_{p}}{L_{q}} \omega i_{d}-\frac{\Delta R_{s}}{L_{q}} i_{q}-\frac{\Delta L_{q}}{L_{q}} \frac{d i_{q}}{d t}-\frac{n_{p} \Delta \phi_{v}}{L_{q}} \omega,
\end{gathered}
$$

where $T_{L}$ the load torque, and

$$
\begin{gathered}
\Delta R_{s}=R_{s t}-R_{s}, \Delta L_{d}=L_{d t}-L_{d}, \Delta L_{q}=L_{q t}-L_{q}, \\
\Delta \phi_{v}=\phi_{v t}-\phi_{v}, \Delta B=B_{t}-B, \Delta J=J_{t}-J,
\end{gathered}
$$

$R_{s t}, L_{d t}, L_{q t}, \phi_{v t}, B_{t}$, and $J_{t}$ denote the actual values of the above mentioned parameters.

Let $x=\left[\omega, i_{d}, i_{q}\right]^{T}$ the state vector, $u=\left[u_{d}, u_{q}\right]^{T}$ the input vector, $w=\left[d_{\omega}, d_{i_{d}}, d_{i_{q}}\right]^{T}$ the disturbance vector, and $y=\left[\omega, i_{d}\right]^{T}$ the output vector, the mathematical model of the PMSM (36) can be represented by the compact form in (2), with

$$
f(x)=\left[\begin{array}{c}
\frac{K_{t}}{J} i_{q}-\frac{B}{J} \omega \\
-\frac{\dot{R} s}{L_{d}} i_{d}+n_{p} \omega i_{q} \\
-\frac{R_{s}}{L_{q}} i_{q}-n_{p} \omega i_{d}-\frac{n_{p} \phi_{v}}{L_{q}} \omega
\end{array}\right], g(x)=\left[\begin{array}{cc}
0 & 0 \\
\frac{1}{L_{d}} & 0 \\
0 & \frac{1}{L_{q}}
\end{array}\right], p(x)=I_{3 \times 3}, h(x)=\left[\begin{array}{c}
h_{1}(x) \\
h_{2}(x)
\end{array}\right]=\left[\begin{array}{c}
\omega \\
i_{d}
\end{array}\right] .
$$

According to the definition of relative degrees for nonlinear systems Isidori (1995), the input relative degrees and disturbance relative degrees of the PMSM system are calculated as $\left(\sigma_{1}, \sigma_{2}\right)=$ $(2,1)$, and $\left(\nu_{1}, \nu_{2}\right)=(1,1)$, respectively. This is the case where the disturbances are mismatched ones.

The parameters of the PMSM under consideration are given as follows: rated power $P=750$ $\mathrm{W}$, rated voltage $U=200 \mathrm{~V}$, rated current $I_{N}=4.71 \mathrm{~A}$, number of pole paries $n_{p}=4$, armature resistance $R_{s}=1.74 \Omega$, stator inductances $L_{d}=L_{q}=0.004 \mathrm{H}$, viscous damping $B=7.403 \times 10^{-5} \mathrm{~N} \cdot \mathrm{m} \cdot \mathrm{s} / \mathrm{rad}$, moment of inertia $J=1.74 \times 10^{-4} \mathrm{Kg} \cdot \mathrm{m}^{2}$, rated speed $3000 \mathrm{rpm}$, rotor flux $\phi_{v}=0.1167 \mathrm{wb}$, rated torque $T_{N}=2.0 \mathrm{~N} \cdot \mathrm{m}$.

In order to show the effectiveness of the proposed method in controlling the PMSM, both the baseline and integral controllers are employed in the simulation studies for performance comparison analysis. The control parameters of the proposed method for the PMSM system are designed as $c_{0}^{1}=44000, c_{1}^{1}=380, c_{0}^{2}=700$. The disturbance observer gain matrix is designed as $\lambda(x)=\left[500 x_{1}, 500 x_{2}, 500 x_{3}\right]$. The baseline controller is given by (33), where the control parameters are designed the same as the proposed method. The integral controller is given by (34), where the integral coefficients are chosen as $c_{i}^{1}=1600000$ and $c_{i}^{2}=120000$, and the rest control parameters are selected the same as the proposed method.

\subsubsection{Tracking performance under unknown load torque variation}

The speed tracking performance is tested under unknown load torque variations in this part. The unknown load torque $T_{L}=2 \mathrm{~N} \cdot \mathrm{m}$ is supposed to add on the PMSM at $t=0.2 \mathrm{sec}$. Response 
(a)
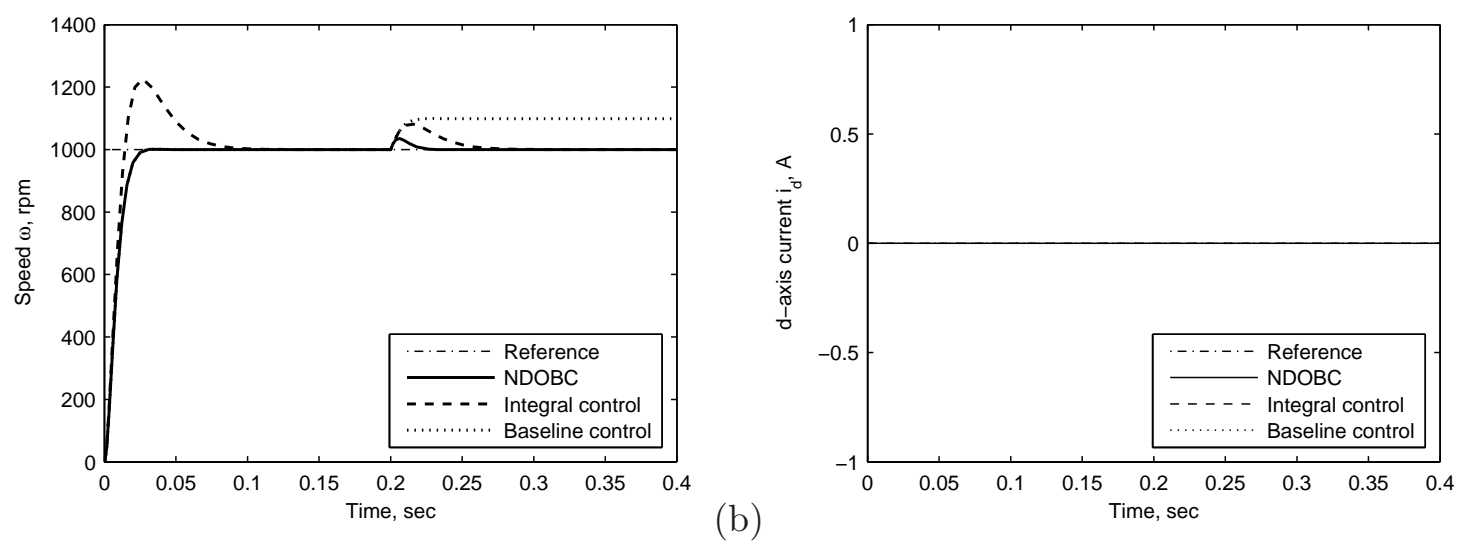

(b)

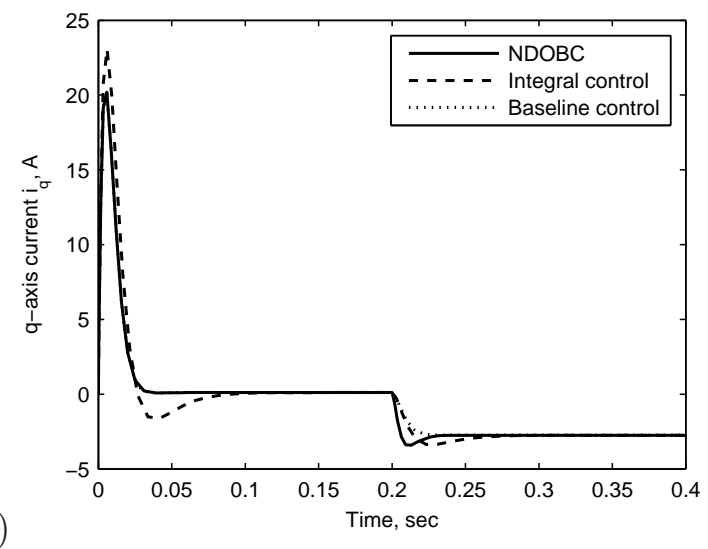

Figure 3. Variable response curves of the PMSM in the presence of unknown load toque variation under the control laws (19) (solid line), (33) (dotted line) and (34) (dashed line). The reference signals are denoted by dash-dotted line.

curves of the rotor speed, $d$-axis and $q$-axis currents are shown in Fig. 3.

As shown by Fig. 3, compared with the integral and baseline control methods, the proposed NDOBC method obtains fine tracking performance in the presence of unknown external load torque variations. It is obtained from Fig. 3 that the response curves of the proposed method are overlaped with the baseline controller during the first $0.2 \mathrm{sec}$ when there is no disturbance, which implies the nominal performance recovery of the proposed method. It is also noticed that the integral control leads to undesirable transient control performance although it can realize offset-free tracking in the presence of load torque variation.

\subsubsection{Tracking performance under uncertain parameters}

To show the robustness of the proposed control approach, the parameters are supposed to have variations from their nominal operation values. Two cases of parameter variations are considered here.

Case I: the stator resistance, the stator inductances, the rotor flux, the moment of inertia and the viscous friction coefficient are supposed to have perturbations $\Delta R_{s}=-0.2 R_{s}, \Delta L_{q}=-0.2 L_{q}$, $\Delta L_{d}=-0.2 L_{d}, \Delta \phi_{v}=0.2 \phi_{v}, \Delta J=-0.2 J$ and $\Delta B=-0.2 B$ respectively.

The response curves of the PMSM under the proposed method in the presence of parameter variations in Case I is shown in Fig. 4.

It can be observed from Fig. 4 that the proposed method gains fine robustness performance in such case of parameter perturbations.

Case II: the stator resistance, the stator inductances, the rotor flux, the moment of inertia and the viscous friction coefficient are supposed to have perturbations $\Delta R_{s}=0.2 R_{s}, \Delta L_{q}=0.2 L_{q}$, $\Delta L_{d}=0.2 L_{d}, \Delta \phi_{v}=-0.2 \phi_{v}, \Delta J=0.2 J$ and $\Delta B=0.2 B$ respectively.

The response curves of the PMSM under the proposed method in the presence of parameter variations in Case II is shown in Fig. 5. 
(a)

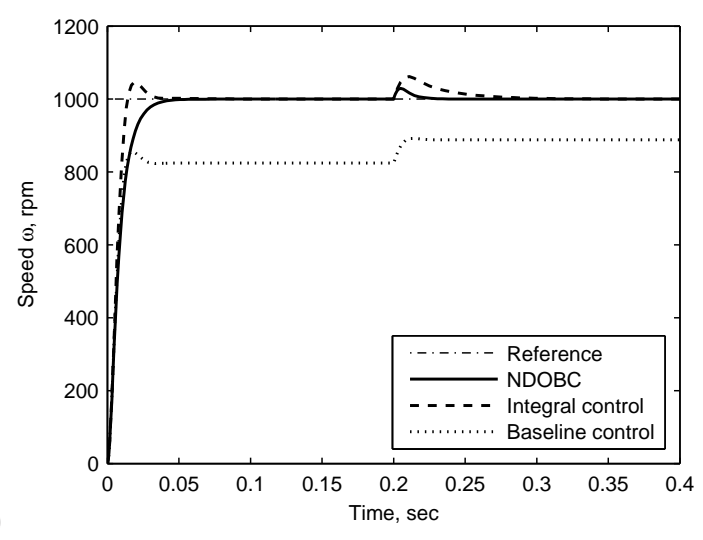

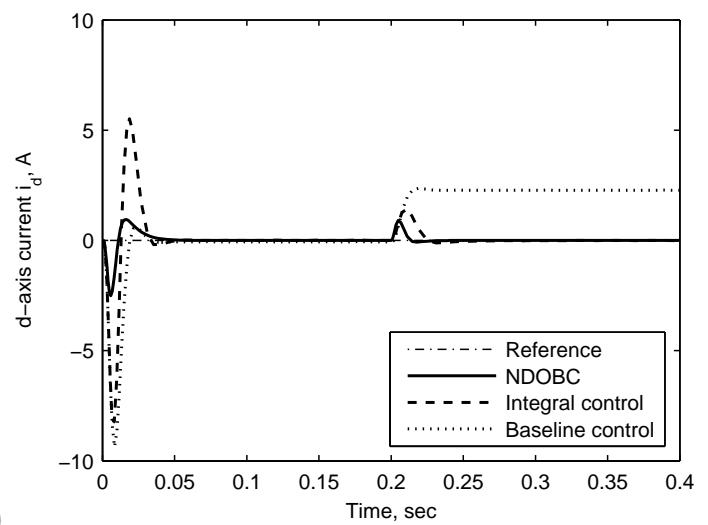

(b)

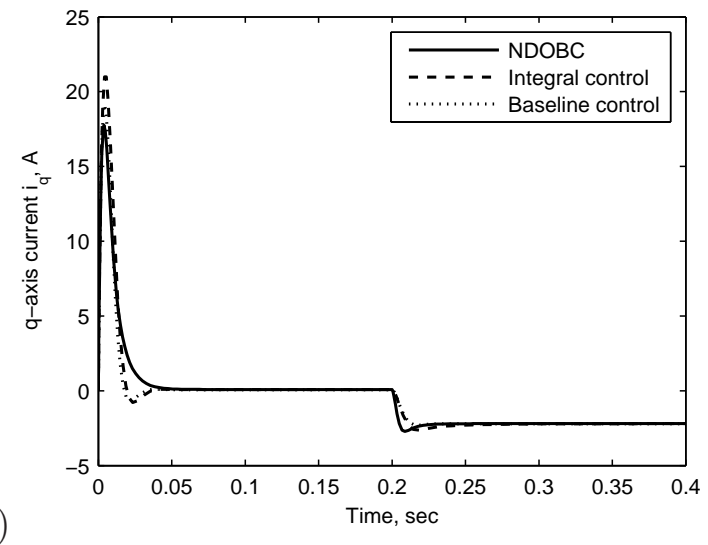

(c)

Figure 4. Variable response curves of the PMSM in the presence of the first case of parameter variations (Case I) under the control laws (19) (solid line), (33) (dotted line) and (34) (dashed line). The reference signals are denoted by dash-dotted line.

The prominent robustness of the proposed method in the second case of parameter variations can be seen from Fig. 5 .

\section{Conclusion}

The disturbance attenuation problem of a MIMO nonlinear system with arbitrary DRD via a DOBC approach, which has been recognized as a longstanding unsolved problem in the field of DOBC, has been solved in this article by designing a new disturbance compensation gain matrix in the control law. It has been proved that the disturbances in this case could be eliminated from the output channels by the newly proposed NDOBC with properly chosen control parameters. The proposed NDOBC method has retained the major feature of the DOBC method, i.e., the ability of recovering its nominal control performance, which has been verified by simulation studies of both numerical and application examples.

\section{Acknowledgements}

The work was supported in part by National 863 Project of the Twelfth Five-Year Plan of China under Grant 2011AA04A106, National Natural Science Foundation of China under Grant 61074013, and Program for New Century Excellent Talents in University under Grant NCET$10-0328$. 
(a)

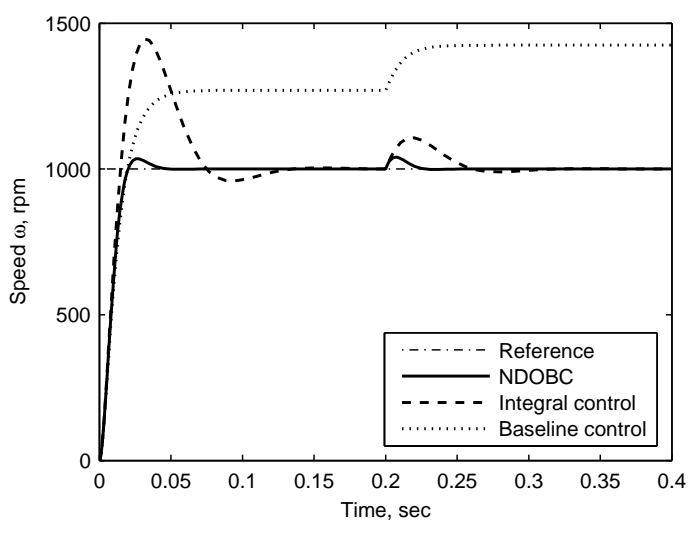

(b)

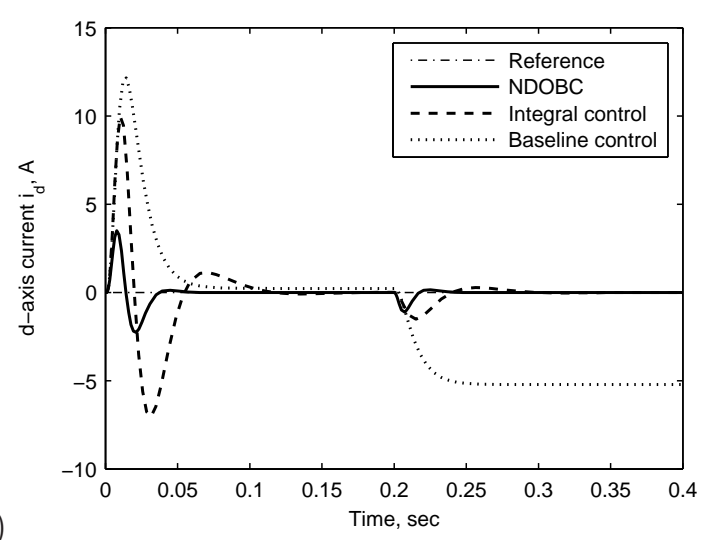

(c)

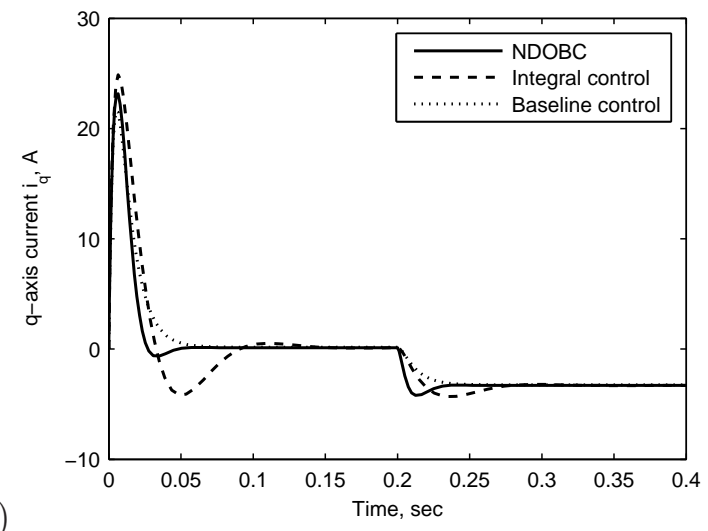

Figure 5. Variable response curves of the PMSM in the presence of the second case of parameter variations (Case II) under the control laws (19) (solid line), (33) (dotted line) and (34) (dashed line). The reference signals are denoted by dash-dotted line.

\section{References}

Aldeen, M., and Sharma, R. (2008), 'Estimation of states, faults and unknown disturbances in non-linear systems', International Journal of Control, 81, 2008, 1195-1201.

Back, J., and Shim, H. (2008), 'Adding robustness to nominal output-feedback controllers for uncertain nonlinear systems: A nonlinear version of disturbance observer', Automatica, 44, $2528-2537$.

Back, J., and Shim, H. (2009), 'An inner-loop controller guaranteeing robust transient performance for uncertain MIMO nonlinear system', IEEE Transactions on Automatic Control, 54, 1601-1607.

Barmish, B.R., and Leitmann, G. (1982), 'On ultimate boundedness control of uncertain systems in the absence of matching assumptions', IEEE Transactions on Automatic Control, 27, 153158.

Chen, W.-H. (2003), 'Nonlinear disturbance observer-enhanced dynamic inversion control of missiles', Journal of Guidance Control and Dynamics, 26, 161-166.

Chen, W.-H., Ballance, D.J., Gawthrop P.J., and O'Reilly, J. (2000), 'A nonlinear disturbance observer for robotic manipulators', IEEE Transactions on Industrial Electronics, 47, 932938.

Chen, W.-H., and Guo, L. (2004), 'Analysis of disturbance observer based control for nonlinear systems under disturbances with bounded variation', In Proceedings of International Conference on Control, Bath, England.

Chen, W.-H., Ballance, D.J., Gawthrop P.J., Gribble, J.J., and O'Reilly, J. (1999), 'Nonlinear PID predictive controller', IEE Proceedings-Control Theory and Applications, 146, 603-611.

Chen, X.K., Komada, S., and Fukuda, T. (2000), 'Design of a nonlinear disturbance observer', 
IEEE Transactions on Industrial Electronics, 47, 429-436.

Fujisaki, Y., and Befekadu, G.K. (2009), 'Reliable decentralised stabilisation of multi-channel systems: a design method via dilated LMIs and unknown disturbance observers', International Journal of Control, 82, 2009, 2040-2050.

Guo, L., and Chen, W.-H. (2005), 'Disturbance attenuation and rejection for systems with nonlinearity via DOBC approach', International Journal of Robust and Nonlinear Control, $15,109-125$.

Han, J. Q. (2009), 'From PID to active disturbance rejection control,' IEEE Transactions on Industrial Electronics, 56, 900-906.

Isidori, A. (1995), Nonlinear Control Systems(3rd ed.), New York: Springer-Verlag.

Isidori, A. (1999), Nonlinear Control Systems: II, New York: Springer-Verlag.

Khalil, H. K. (1996), Nonlinear Systems(2nd ed.), Upper Saddle River, N.J.: Prentice-Hall.

Krishnan,R. (2001) Electric Motor Drives: Modeling, Analysis, and Control, Upper Saddle River, N.J.: Prentice-Hall.

Li, S.H., Yang, J., Chen, W.-H., and Chen, X.S. (2012), 'Generalized extended state observer based control for systems with mismatched uncertainties,' IEEE Transactions on Industrial Electronics, available online.

Maeder, U, and Morari, M. (2010), 'Offset-free reference tracking with model predictive control', Automatica, 46(9), 1469-1476.

Marino, R., Respondek, W., and V.D. Schaft, A.J. (1989), 'Almost disturbance decoupling for single-input single-output nonlinear systems', IEEE Transactions on Automatic Control, 34(9), 1013-1017.

Mohamed, Y.A.-R.I. (2007), 'Design and implementation of a robust current-control scheme for a PMSM vector drive with a simple adaptive disturbance observer', IEEE Transactions on Industrial Electronics, 54, 1981-1988.

She, J.-H., Fang, M., Ohyama, Y., Hashimoto, H., and Wu, M. (2008), 'Improving disturbancerejection performance based on an equivalent-input-disturbance approach', IEEE Transactions on Industrial Electronics, 55, 380-389.

Shim, H., and Jo, N.H. (2009), ' An almost necessary and sufficient condition for robust stability of closed-loop systems with disturbance observer', Automatica, 45, 296-299.

Wei, X.J., and Guo, L. (2009), 'Composite disturbance-observer-based control and terminal sliding mode control for non-linear systems with disturbances', International Journal of Control, 82, 2009, 1082-1098.

Wei, X.J., and Guo, L. (2010), 'Composite disturbance-observer-based control and H-infinity control for complex continuous models', International Journal of Robust and Nonlinear Control, 20, 106-118.

Wonham, W.M. (1985), Linear Multivariable Control: A Geometric Approach(3rd ed.), New York: Springer-Verlag.

Xia, Y., Shi, P., Liu, G. P., Rees, D., and Han, J. (2007), 'Active disturbance rejection control for uncertain multivariable systems with time-delay,' IET Control Theory and Applications, $1,75-81$.

Xia, Y., Zhu, Z., Fu, M., and Wang, S. (2011), 'Attitude tracking of rigid spacecraft with bounded disturbances,' IEEE Transactions on Industrial Electronics, 58, 647-659.

Yang, J., Chen, W.-H., and Li, S.H. (2011), 'Nonlinear disturbance observer based control for systems with mismatched disturbances/uncertainties', IET Control Theory and Applications, 5, 2053-2062.

Yang, J., Li, S.H., and Yu, X. (2012), 'Sliding-mode control for systems with mismatched uncertainties via a disturbance observer,' IEEE Transactions on Industrial Electronics, available online.

Yang, J., Zolotas, A., Chen, W.-H., Michail, K., and Li, S.H. (2011), 'Robust control of nonlinear MAGLEV suspension system with mismatched uncertainties via DOBC approach', ISA Transactions, 50, 389-396. 
Yoo, D., Yau, S.S.-T., and Gao, Z. (2007), 'Optimal fast tracking observer bandwidth of the
linear extended state observer', International Journal of Control, 80, 2007, 102-111.

Yoo, D., Yau, S.S.-T., and Gao, Z. (2007), 'Optimal fast tracking observer bandwidth
linear extended state observer', International Journal of Control, 80, 2007, 102-111.

\begin{abstract}
linear extended state observer', International Journal of Control, 80, 2007, 102-111.
\end{abstract}

